\title{
Association of ALDH3B2 gene polymorphism and risk factors with susceptibility of esophageal squamous cell carcinoma in a Chinese population: a case-control study involving 2,358 subjects
}

\author{
Jun Yin 1,2, ${ }^{1}$, Weifeng Tang ${ }^{1, *}$, Tao Long ${ }^{1, *}$, Huiwen Pan ${ }^{1}$, Jianchao Liu ${ }^{1}$, Lu Lv ${ }^{1}$, Chao Liu ${ }^{1}$, \\ Yijun Shi ${ }^{1}$, Jingfeng Zhu ${ }^{1}$, Yangyong Sun ${ }^{1}$, Aizhong Shao ${ }^{1}$, Qiang Zhou ${ }^{1}$, Zhengbing \\ Ren ${ }^{1}$, Guowen Ding ${ }^{1}$, Suocheng Chen ${ }^{1}$, Yan Liư ${ }^{3}$, Jun Yao ${ }^{4}$, Hao Ding ${ }^{5}$, Yulan Yan ${ }^{5}$, \\ Haiyong Gu${ }^{6}$, Cheng Qian², Liming Wang7, Qun Wang² and Lijie Tan² \\ ${ }^{1}$ Department of Cardiothoracic Surgery, Affiliated People's Hospital of Jiangsu University, Zhenjiang, Jiangsu, 212002, China \\ ${ }^{2}$ Department of Thoracic Surgery, Zhongshan Hospital of Fudan University, Shanghai, 200032, China \\ ${ }^{3}$ Genesky Biotechnologies Inc., Shanghai, 201315, China \\ ${ }^{4}$ Department of Gastroenterology, Affiliated People's Hospital of Jiangsu University, Zhenjiang, Jiangsu, 212002, China \\ ${ }^{5}$ Department of Respirology, Affiliated People's Hospital of Jiangsu University, Zhenjiang, Jiangsu, 212002, China \\ ${ }^{6}$ Department of Thoracic Surgery, Shanghai Chest Hospital, Shanghai Jiaotong University, Shanghai, 200030, China \\ ${ }^{7}$ Cancer Institute, Department of Chemotherapy, Affiliated People's Hospital of Jiangsu University, Zhenjiang, Jiangsu, 212002, China \\ "These authors contributed equally to this work
}

Correspondence to: Liming Wang, email: yin912@outlook.com

Qun Wang, email: wang.qun@zs-hospital.sh.cn

Lijie Tan, email: tan.lijie@zs-hospital.sh.cn

Keywords: ALDH3B2; polymorphisms; risk factors; esophageal squamous cell carcinoma

Received: July 21, $2017 \quad$ Accepted: October 30, $2017 \quad$ Published: November 24, 2017

Copyright: Yin et al. This is an open-access article distributed under the terms of the Creative Commons Attribution License 3.0 (CC BY 3.0), which permits unrestricted use, distribution, and reproduction in any medium, provided the original author and source are credited.

\section{ABSTRACT}

Background: Esophageal cancer (EC) is the sixth leading cause of cancerassociated death worldwide. The interaction of environmental risk factors and genetic factors might contribute to the carcinogenesis of EC synergistically.

Results: All seven single locus polymorphisms of ALDH3B2 were not associated with risk of ESCC as evaluated by allelic, dominant, co-dominant, recessive and Cochran-Armitage trend tests. Stratified analyses showed these SNPs were not correlated with the susceptibility of ESCC according to different age, gender, cigarette smoking and alcohol drinking status. None of the major haplotypes were related with ESCC susceptibility.

Materials and Methods: We conducted a hospital-based case-control study to evaluate the combined effects of environmental risk factors and the single nucleotide polymorphisms (SNPs) of ALDH3B2 gene on the development of esophageal squamous carcinoma (ESCC). A total of 1043 ESCC cases and 1315 controls were recruited for this study. Seven ALDH3B2 SNPs and four environmental factors were selected as independent variables. ALDH3B2 SNPs were determined by ligation detection reaction method.

Conclusions: Our study suggested that ALDH3B2 rs34589365, rs3741172, rs4646823, rs78402723, rs7947978, rs866907 and rs9787887 polymorphisms were not implicated with altered susceptibility of ESCC according to different age, gender, cigarette smoking and alcohol drinking status. Yet this conclusion needs to be verified in larger studies among different ethnic populations with validation design, the biological function of these SNPs in carcinogenesis are subject to further investigation. 


\section{INTRODUCTION}

Esophageal cancer ranks the ninth most common cancer and the sixth most common cause of cancer death worldwide [1]. Despite remarkable advances in the therapeutic strategy, extensive treatment may be associated with a noticeable decline in health-related quality of life and yet a poor prognosis [2]. Approximately $70 \%$ of global esophageal cancer cases occur in China, with esophageal squamous cell carcinoma (ESCC) being the histopathological form in the vast majority of cases (> 90\%) [3]. Alcohol consumption [4, 5], tobacco use $[4,6]$, poor oral hygiene, low socioeconomic status and nutritional deficiencies have been reported risk factors for esophageal cancer [7-10]. The fact that only a subset of cohort that are exposed to the risk factors eventually develop esophageal cancer suggested a critical role of genetic factors, including single nucleotide polymorphisms (SNPs), in the esophageal carcinogenesis.

Among all risk factors, others and we have repeatedly verified the indisputable role of alcohol consumption in the ESCC carcinogenesis [11, 12]. Extensive evaluations and reviews of alcohol-related cancers have been published in the Monographs of the International Agency of Research on Cancer (IARC) $[13,14]$, with most convincing evidence (Group I) for alcohol-drinking-related cancers is targeted on esophagus and some other organs [15]. Specific enzymes, whose activity and expression are influenced by genetic polymorphisms, regulate the metabolism of alcohol [11]. In humans, alcohol is primarily metabolized by two major groups of enzymes termed alcohol dehydrogenases (ADHs) and aldehyde dehydrogenases (ALDHs) [16]. In the cytosol of hepatocytes, ADHs catalyse the oxidation of ethanol to acetaldehyde, which is further oxidized to acetate by ALDHs in the mitochondria [11]. Although alcohol is not a carcinogen per se, its metabolite acetaldehyde is a toxin and carcinogen that rapidly binds to protein and DNA. It has profound effects on carcinogenesis by forming with DNA carcinogenic DNA adducts, by inhibiting DNA repair and by regulating DNA methylation. Acetaldehyde is degradated by ALDHs, which renders ALDHs a pivotal role in the carcinogenesis. Indeed, high ALDH1 expression predicts unfavorable outcomes in patients with ESCC [17]. Individuals with $A L D H 2$ Lys allele possess a higher risk of esophageal cancer, in correlation with a higher concentration of blood acetaldehyde after drinking alcohol [18]. There is a strong association between $A L D H 2$ Glu487Lys polymorphism and the risk of esophageal cancer [19]. ALDH2 rs671 [20] and rs886205 [21] polymorphisms have also been demonstrated to correlate with ESCC, respectively.

Similarly, the aldehyde dehydrogenase 3 family member B2 (ALDH3B2) is also a key member of ALDH family. Originally identified as ALDH8, ALDH3B2 encodes a member of the aldehyde dehydrogenase family, a group of isozymes that may play a major role in the detoxification of aldehydes generated by alcohol metabolism and lipid peroxidation. As compared with ALDH1/2, little is known on ALDH3 family (including ALDH3B2) with respect to their roles in carcinogenesis. The association between ALDH3B2 polymorphisms and ESCC has not been investigated. Hence, in this hospitalbased case-control study, we performed genotyping analyses of the seven SNPs in 1043 ESCC cases and 1315 controls in a Chinese population.

\section{RESULTS}

\section{Characteristics of the study subjects}

The characteristics of the study subjects, including the demographics and environmental risk factors, are presented in Table 1. The controls and cases were well matched in age and gender ( $\chi^{2}$ test, $p=0.121$ and 0.880 , respectively). However, the cigarette-smoking rate $(43.53 \%$ vs. $26.70 \%, p<0.001)$ and alcohol drinking rate $(31.54 \%$ vs. $7.07 \%, p<0.001)$ were both significantly higher in the ESCC cases.

As shown in Table 2, the genotyping successful rates were all beyond $98.81 \%$. In the control subjects, the genotype frequencies for the seven polymorphisms reached Hardy-Weinberg equilibrium ( $p$-value for HWE, all $p>0.05$, Table 2).

\section{Associations between risk of ESCC and seven polymorphisms}

As shown in Table 3, the single locus analyses showed no statistically significant difference in genotype frequencies of seven SNPs between the cases and controls $(p>0.05)$. As assessed by the allelic, dominant, co-dominant, recessive and Cochran-Armitage trend tests, there are no correlations between these seven polymorphism sites with the risk of ESCC (Table 3).

\section{Stratification analyses on seven polymorphisms and risk of ESCC}

To further evaluate the effects of $A L D H 3 B 2$ rs34589365, rs3741172, rs4646823, rs78402723, rs7947978, rs866907 and rs9787887 on ESCC risk with different gender, age, smoking and alcohol drinking status, stratification analyses were performed as demonstrated in the Tables 4-10.

Our analyses demonstrated that neither gender, age, cigarette smoking nor alcohol drinking has detectable impacts on the susceptibility of ESCC after stratified analyses. 
Table 1: Distribution of selected demographic variables and risk factors in ESCC cases and controls

\begin{tabular}{|c|c|c|c|c|c|}
\hline \multirow{2}{*}{ Variable } & \multicolumn{2}{|l|}{ Cases $(n=1043)$} & \multicolumn{2}{|c|}{ Controls $(n=1315)$} & \multirow{2}{*}{$p$} \\
\hline & $n$ & $\%$ & $n$ & $\%$ & \\
\hline Age (years) mean \pm SD & \multicolumn{2}{|l|}{$63.07( \pm 7.27)$} & \multicolumn{2}{|c|}{$62.88( \pm 9.74)$} & 0.607 \\
\hline \multicolumn{6}{|l|}{ Age (years) } \\
\hline$<63$ & 471 & 45.16 & 636 & 48.37 & \\
\hline$\geq 63$ & 572 & 54.84 & 679 & 51.63 & 0.121 \\
\hline \multicolumn{6}{|l|}{ Sex } \\
\hline Male & 758 & 72.67 & 952 & 72.40 & \\
\hline Female & 285 & 27.33 & 363 & 27.63 & 0.880 \\
\hline \multicolumn{6}{|l|}{ Tobacco use } \\
\hline Never & 589 & 56.47 & 964 & 73.30 & \\
\hline Ever & 454 & 43.53 & 351 & 26.70 & $<0.001$ \\
\hline \multicolumn{6}{|l|}{ Alcohol use } \\
\hline Never & 714 & 68.46 & 1222 & 92.93 & \\
\hline Ever & 329 & 31.54 & 93 & 7.07 & $<0.001$ \\
\hline
\end{tabular}

${ }^{\text {a }}$ Two-sided $\chi^{2}$ test and student $t$ test; Bold values are statistically significant $(p<0.05)$.

Table 2: Primary information for $A L D H 3 B 2$ rs34589365, rs3741172, rs4646823, rs78402723, rs7947978, rs866907, rs9787887 polymorphisms

\begin{tabular}{|c|c|c|c|c|c|c|c|}
\hline $\begin{array}{l}\text { Genotyped } \\
\text { SNPs }\end{array}$ & rs34589365 & rs3741172 & rs4646823 & rs78402723 & rs7947978 & rs866907 & rs9787887 \\
\hline Ancestral Allele & $\mathrm{A}$ & G & G & G & $\mathrm{C}$ & $\mathrm{A}$ & G \\
\hline Chromosome & 11 & 11 & 11 & 11 & 11 & 11 & 11 \\
\hline Gene (ID) & ALDH3B2(222) & ALDH3B2(222) & ALDH3B2(222) & ALDH3B2(222) & ALDH3B2(222) & ALDH3B2(222) & ALDH3B2(222) \\
\hline Function & intron-variant & $\begin{array}{c}\text { reference, } \\
\text { synonymous-codon }\end{array}$ & $\begin{array}{l}\text { utr-variant-5- } \\
\text { prime }\end{array}$ & $\begin{array}{l}\text { utr-variant-3- } \\
\text { prime }\end{array}$ & $\begin{array}{l}\text { intron-variant, } \\
\text { utr-variant-5- } \\
\text { prime }\end{array}$ & $\begin{array}{l}\text { utr-variant-3- } \\
\text { prime }\end{array}$ & intron-variant \\
\hline $\begin{array}{c}\text { Chr Pos } \\
\text { (Genome Build } \\
\text { 38.p7) }\end{array}$ & 67668505 & 67663227 & 67666945 & 67663118 & 67674572 & 67663011 & 67673240 \\
\hline $\begin{array}{l}\text { Regulome DB } \\
\text { Score }^{\mathrm{a}}\end{array}$ & 5 & 5 & 4 & 4 & 4 & 4 & 5 \\
\hline TFBS $^{\mathrm{b}}$ & $\mathrm{Y}$ & -- & Y & -- & -- & -- & -- \\
\hline nsSNP & -- & -- & -- & -- & -- & -- & -- \\
\hline $\begin{array}{l}\mathrm{MAF}^{\mathrm{c}} \text { for } \\
\text { Chinese in } \\
\text { database }\end{array}$ & 0.183 & -- & 0.098 & 0.058 & 0.098 & 0.128 & -- \\
\hline $\begin{array}{l}\text { MAF in our } \\
\text { controls } \\
(\boldsymbol{n}=1315)\end{array}$ & 0.126 & 0.084 & 0.105 & 0.084 & 0.105 & 0.105 & 0.335 \\
\hline $\begin{array}{l}p \text { value for } \\
\text { HWE } E^{d} \text { test in our } \\
\text { controls }\end{array}$ & 0.110 & 0.784 & 0.294 & 0.808 & 0.279 & 0.294 & 0.258 \\
\hline $\begin{array}{l}\text { Genotyping } \\
\text { method }^{\mathrm{e}}\end{array}$ & LDR & LDR & LDR & LDR & LDR & LDR & LDR \\
\hline $\begin{array}{l}\% \text { Genotyping } \\
\text { value }\end{array}$ & $99.02 \%$ & $99.02 \%$ & $99.02 \%$ & $98.98 \%$ & $99.02 \%$ & $99.02 \%$ & $98.81 \%$ \\
\hline
\end{tabular}

${ }^{a}$ http://www.regulomedb.org/;

'TFBS: Transcription Factor Binding Site (https://snpinfo.niehs.nih.gov/cgi-bin/snpinfo/snpfunc.cgi);

'MAF: minor allele frequency;

${ }^{\mathrm{d}}$ HWE: Hardy-Weinberg equilibrium;

eLDR: ligation detection reaction. 
Table 3: Main effects of $A L D H 3 B 2$ SNPs on ESCC risk

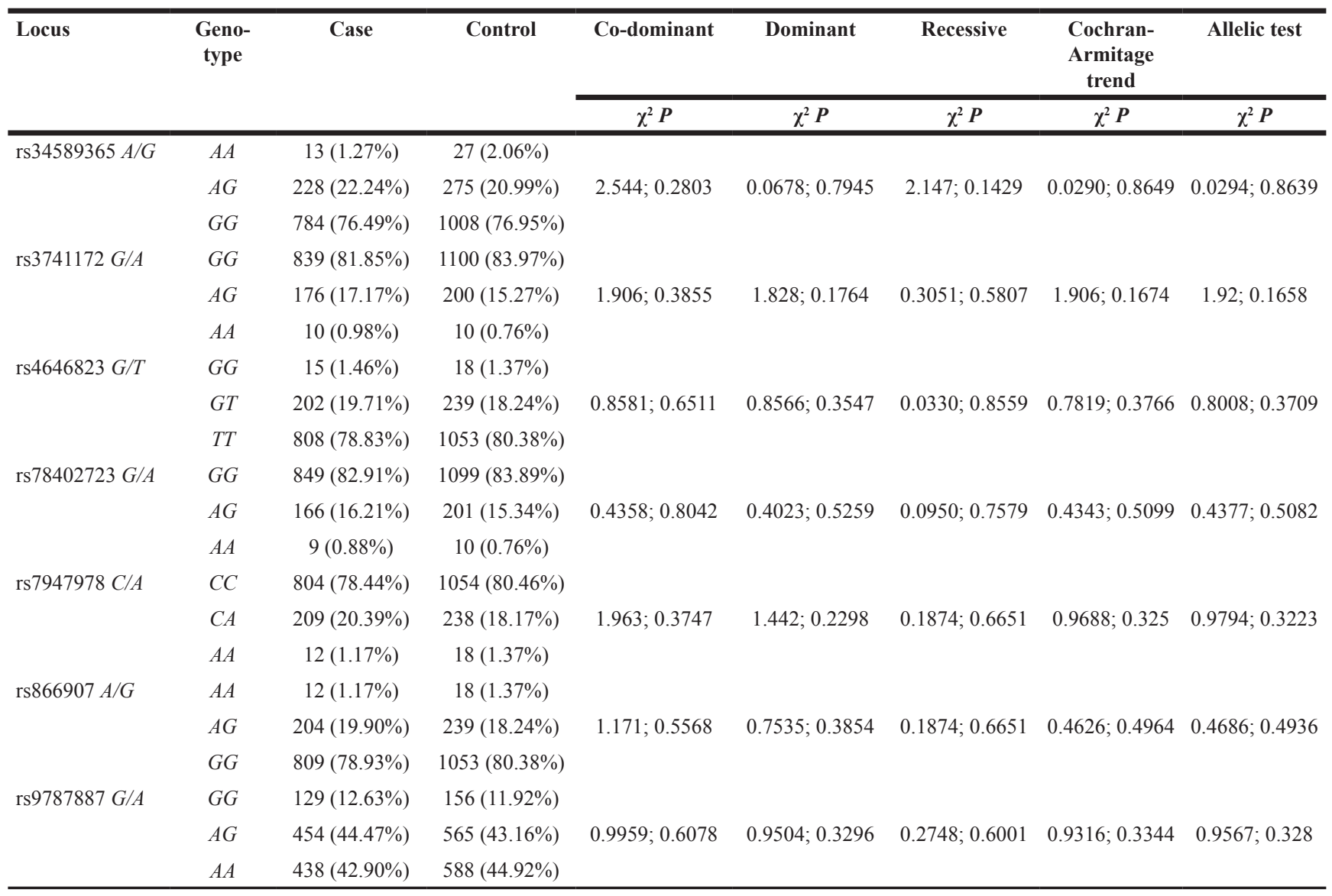

Table 4: Stratified analyses between $A L D H 3 B 2$ rs34589365 polymorphism and ESCC risk by sex, age, smoking status and alcohol consumption

\begin{tabular}{|c|c|c|c|c|c|c|c|c|c|}
\hline \multirow{2}{*}{ Variable } & \multicolumn{4}{|c|}{ (case/control) $^{\mathrm{a}}$} & \multicolumn{5}{|c|}{ Adjusted $\mathrm{OR}^{\mathrm{b}}(95 \% \mathrm{CI}) ; p$} \\
\hline & AA & GG & AG & $\mathbf{A G}+\mathbf{G G}$ & $\mathbf{A A}$ & GG & AG & AG+GG & GG vs. $(A A+A G)$ \\
\hline \multicolumn{10}{|l|}{ Sex } \\
\hline Male & $12 / 19$ & $554 / 717$ & $179 / 212$ & $733 / 929$ & 1.00 & $\begin{array}{c}0.817(0.39-1.70) \\
p: 0.588\end{array}$ & $\begin{array}{c}0.748(0.35-1.58) \\
p: 0.446\end{array}$ & $\begin{array}{c}0.800(0.39-1.66) \\
p: 0.547\end{array}$ & $\begin{array}{c}0.934(0.75-1.17) \\
p: 0.549\end{array}$ \\
\hline Female & $1 / 8$ & $230 / 291$ & $49 / 63$ & $279 / 354$ & 1.00 & $\begin{array}{c}1.158(0.02-1.27) \\
p: 0.085\end{array}$ & $\begin{array}{c}0.161(0.02-1.33) ; \\
p: 0.079\end{array}$ & $\begin{array}{c}0.159(0.02-1.28) \\
p: 0.085\end{array}$ & $\begin{array}{c}1.122(0.75-1.68) \\
p: 0.573\end{array}$ \\
\hline \multicolumn{10}{|l|}{ Age } \\
\hline$<63$ & $5 / 14$ & $347 / 478$ & $114 / 140$ & $461 / 618$ & 1.00 & $\begin{array}{c}0.492(0.18-1.38) \\
p: 0.239\end{array}$ & $\begin{array}{c}0.439(0.15-1.25) ; \\
p: 0.151\end{array}$ & $\begin{array}{c}0.479(0.17-1.34) \\
p: 0.168\end{array}$ & $\begin{array}{c}0.939(0.71-1.24) \\
p: 0.658\end{array}$ \\
\hline$\geq 63$ & $8 / 13$ & $437 / 530$ & $114 / 135$ & $551 / 665$ & 1.00 & $\begin{array}{c}0.746(0.31-1.82) \\
p: 0.659\end{array}$ & $\begin{array}{c}0.729(0.29-1.82) \\
p: 0.497\end{array}$ & $\begin{array}{c}0.746(0.31-1.82) \\
p: 0.518\end{array}$ & $\begin{array}{c}1.000(0.76-1.31) \\
p: 0.999\end{array}$ \\
\hline \multicolumn{10}{|l|}{ Smoking status } \\
\hline Never & $8 / 21$ & $449 / 743$ & $117 / 196$ & $566 / 939$ & 1.00 & $\begin{array}{c}0.630(0.28-1.44) \\
p: 0.268\end{array}$ & $\begin{array}{c}0.638(0.27-1.49) \\
p: 0.295\end{array}$ & $\begin{array}{c}0.632(0.28-1.44) \\
p: 0.269\end{array}$ & $\begin{array}{c}1.049(0.82-1.35) \\
p: 0.706\end{array}$ \\
\hline Ever & $5 / 6$ & $335 / 265$ & $111 / 79$ & $446 / 344$ & 1.00 & $\begin{array}{c}0.659(0.20-2.18) \\
p: 0.550\end{array}$ & $\begin{array}{c}0.593(0.18-2.01) \\
p: 0.533\end{array}$ & $\begin{array}{c}0.643(0.20-2.12) \\
p: 0.547\end{array}$ & $\begin{array}{c}0.926(0.67-1.28) \\
p: 0.642\end{array}$ \\
\hline \multicolumn{10}{|c|}{ Alcohol consumption } \\
\hline Never & $10 / 26$ & $555 / 945$ & $138 / 246$ & $693 / 1191$ & 1.00 & $\begin{array}{c}0.655(0.31-1.37) \\
p: 0.257\end{array}$ & $\begin{array}{c}0.686(0.32-1.46) \\
p: 0.327\end{array}$ & $\begin{array}{c}0.661(0.32-1.38) ; \\
p: 0.267\end{array}$ & $\begin{array}{c}1.079(0.86-1.35) \\
p: 0.508\end{array}$ \\
\hline Ever & $3 / 1$ & $229 / 63$ & $90 / 29$ & $319 / 92$ & 1.00 & $\begin{array}{c}0.825(0.08-8.07) ; \\
p: 1.000\end{array}$ & $\begin{array}{c}0.967(0.10-9.66) ; \\
p: 1.000\end{array}$ & $\begin{array}{c}0.865(0.09-8.42) \\
p: 1.000\end{array}$ & $\begin{array}{c}1.173(0.71-1.93) \\
p: 0.530\end{array}$ \\
\hline
\end{tabular}

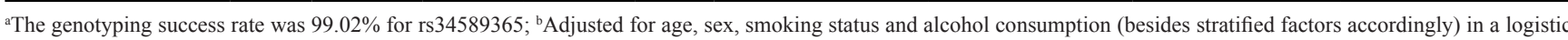
regression model; Fisher exact test was performed when $n \leq 5$; Bonferroni correction was performed to correct the $p$ value, bold values are statistically significant $(p<0.0125)$. 
Table 5: Stratified analyses between $A L D H 3 B 2$ rs3741172 polymorphism and ESCC risk by sex, age, smoking status and alcohol consumption

\begin{tabular}{|c|c|c|c|c|c|c|c|c|c|}
\hline \multirow{2}{*}{ Variable } & \multicolumn{4}{|c|}{$(\text { case/control) })^{a}$} & \multicolumn{5}{|c|}{ Adjusted OR b $(95 \% \mathrm{CI}) ; p$} \\
\hline & GG & $\mathbf{A A}$ & $\mathbf{A G}$ & $\mathbf{A A}+\mathbf{A G}$ & GG & $\mathbf{A A}$ & $\mathbf{A G}$ & $\mathbf{A A}+\mathbf{A G}$ & AA vs. (GG+AG) \\
\hline \multicolumn{10}{|l|}{ Sex } \\
\hline Male & $622 / 798$ & $5 / 7$ & $118 / 143$ & $123 / 150$ & 1.00 & $\begin{array}{c}1.091(0.35-3.46) \\
p: 1.000\end{array}$ & $\begin{array}{c}0.945(0.72-1.23) \\
p: 0.674\end{array}$ & $\begin{array}{c}0.951(0.73-1.23) ; \\
p: 0.703\end{array}$ & $\begin{array}{c}0.908(0.29-2.87) ; \\
p: 1.000\end{array}$ \\
\hline Female & $217 / 302$ & $5 / 3$ & $58 / 57$ & $63 / 60$ & 1.00 & $\begin{array}{c}0.431(0.10-1.82) \\
p: 0.291\end{array}$ & $\begin{array}{c}0.706(0.47-1.06) \\
p: 0.091\end{array}$ & $\begin{array}{c}0.684(0.46-1.02) \\
p: 0.059\end{array}$ & $\begin{array}{c}2.176(0.52-9.18) ; \\
p: 0.305\end{array}$ \\
\hline \multicolumn{10}{|l|}{ Age } \\
\hline$<63$ & $387 / 527$ & $4 / 8$ & $75 / 97$ & $79 / 105$ & 1.00 & $\begin{array}{c}1.469(0.44-4.91) \\
p: 0.770\end{array}$ & $\begin{array}{c}0.950(0.68-1.32) \\
p: 0.759\end{array}$ & $\begin{array}{c}0.976(0.71-1.34) \\
p: 0.882\end{array}$ & $\begin{array}{c}0.675(0.20-2.26) \\
p: 0.573\end{array}$ \\
\hline$\geq 63$ & $452 / 573$ & $6 / 2$ & $101 / 103$ & $107 / 105$ & 1.00 & $\begin{array}{c}0.263(0.05-1.31) \\
p: 0.149\end{array}$ & $\begin{array}{c}0.804(0.60-1.09) \\
p: 0.156\end{array}$ & $\begin{array}{c}0.774(0.58-1.04) \\
p: 0.090\end{array}$ & $\begin{array}{c}3.667(0.74-18.24) ; \\
p: 0.151\end{array}$ \\
\hline \multicolumn{10}{|l|}{ Smoking status } \\
\hline Never & $463 / 811$ & $6 / 7$ & $105 / 142$ & $111 / 149$ & 1.00 & $\begin{array}{c}0.666(0.22-1.99) \\
p: 0.465\end{array}$ & $\begin{array}{c}0.772(0.59-1.02) \\
p: 0.067\end{array}$ & $\begin{array}{c}0.766(0.58-1.01) \\
p: 0.054\end{array}$ & $\begin{array}{c}1.438(0.48-4.30) ; \\
p: 0.513\end{array}$ \\
\hline Ever & $376 / 289$ & $4 / 3$ & $71 / 58$ & $75 / 61$ & 1.00 & $\begin{array}{c}0.976(0.22-4.39) \\
p: 1.000\end{array}$ & $\begin{array}{c}1.063(0.73-1.55) \\
p: 0.753\end{array}$ & $\begin{array}{c}1.058(0.73-1.53) ; \\
p: 0.765\end{array}$ & $\begin{array}{c}1.035(0.23-4.66) ; \\
p: 1.000\end{array}$ \\
\hline \multicolumn{10}{|c|}{ Alcohol consumption } \\
\hline Never & $571 / 1024$ & $7 / 9$ & $125 / 184$ & $132 / 193$ & 1.00 & $\begin{array}{c}0.717(0.27-1.94) \\
p: 0.509\end{array}$ & $\begin{array}{c}0.821(0.64-1.05) ; \\
p: 0.120\end{array}$ & $\begin{array}{c}0.815(0.64-1.04) \\
p: 0.100\end{array}$ & $\begin{array}{c}1.350(0.50-3.64) ; \\
p: 0.552\end{array}$ \\
\hline Ever & $268 / 76$ & $3 / 1$ & $51 / 16$ & $54 / 17$ & 1.00 & $\begin{array}{c}1.175(0.12-11.46) ; \\
p: 1.000\end{array}$ & $\begin{array}{c}1.106(0.60-2.05) ; \\
p: 0.748\end{array}$ & $\begin{array}{c}1.110(0.61-2.03) ; \\
p: 0.733\end{array}$ & $\begin{array}{c}0.865(0.09-8.42) ; \\
p: 1.000\end{array}$ \\
\hline
\end{tabular}

${ }^{\text {a }}$ The genotyping success rate was $99.02 \%$ for rs 3741172 ; ${ }^{\mathrm{b}}$ Adjusted for age, sex, smoking status and alcohol consumption (besides stratified factors accordingly) in a logistic regression model; Fisher exact test was performed when $\mathrm{n} \leq 5$; Bonferroni correction was performed to correct the $p$ value, bold values are statistically significant $(p<0.0125)$.

\section{Linkage disequilibrium analyses and association test}

Linkage disequilibrium analyses in both controls and cases were performed as shown in Tables 11,12 and Figures 1, 2. There were strong correlations between these seven loci. Association test was conducted using Haploview software (version 4.2), strong associations were detected between these seven loci.

\section{Haplotype analyses of ALDH3B2 polymorphisms and susceptibility of ESCC}

As demonstrated in Table 13, haplotype analyses showed that ALDH3B2 $\mathrm{G}_{\mathrm{rs} 34589365} \mathrm{G}_{\mathrm{rs} 3741172} \mathrm{~T}_{\mathrm{r} 54646823} \mathrm{G}_{\mathrm{rs} 78402723} \mathrm{C}_{\mathrm{rs} 7947978} \mathrm{G}_{\mathrm{rs} 866907} \mathrm{~A}_{\mathrm{rs} 9787887}$ was most common haplotype in both groups $(66.3 \%$ in controls, $64.6 \%$ in cases). As compared with $\mathrm{G}_{\mathrm{rs} 34589365} \mathrm{G}_{\mathrm{rs} 3741172} \mathrm{~T}_{\mathrm{rs} 46466823} \mathrm{G}_{\mathrm{rs} 78402723} \mathrm{C}_{\mathrm{rs} 5947978} \mathrm{G}_{\mathrm{rs} 866907} \mathrm{~A}_{\mathrm{rs} 9787887}$, none of the haplotypes was associated with the susceptibility of ESCC.

\section{Power calculation}

The power calculation was performed using the "Power and Sample Size Calculation" software (http:// biostat.mc.vanderbilt.edu/wiki/Main/PowerSampleSize). Based on the assumption that the type I error probability for a two sided test $\alpha$ equals 0.05 , the probability of exposure in controls $\mathrm{P}_{0}$ is 0.126 in control. In the current study, using ligation detection reaction method, the successful rate of genotyping exceeded $98 \%$. There were in total 1315 controls and 1043 cases successfully genotyped. The ratio of control/case (m) equals 1.261, and the correlation coefficient for exposure between matched case and controls ( $\mathrm{f}$ ) is 0.619 . The power value is 0.936 as calculated by the "Power and Sample Size Calculation" software.

\section{DISCUSSION}

In this hospital-based case-control epidemiological study, we investigated the association between tagging SNPs of $A L D H 3 B 2$ and the risk of developing esophageal squamous cell carcinoma in a Chinese population. We found $A L D H 3 B 2$ rs 34589365 , rs3741172, rs 4646823 , rs78402723, rs7947978, rs866907 and rs9787887 polymorphisms were not implicated with altered susceptibility of ESCC according to age, gender, cigarette smoking and alcohol drinking stratification analyses.

Despite a suspected association between alcohol drinking and death to cancer reported in an epidemiological study as early as 1903, it took until 1988 for the research community to agree on the potential risk through the International Agency for Research on Cancer (IARC). Clear Patterns have emerged between alcohol consumption and esophageal cancer. Essentially, alcohol and its metabolite acetaldehyde were both designated as type 1A carcinogen [13]. The cytotoxic properties, the ability to form DNA-acetaldehyde adducts and to generate 
Table 6: Stratified analyses between $A L D H 3 B 2$ rs4646823 polymorphism and ESCC risk by sex, age, smoking status and alcohol consumption

\begin{tabular}{|c|c|c|c|c|c|c|c|c|c|}
\hline \multirow{2}{*}{ Variable } & \multicolumn{4}{|c|}{ (case/control) a } & \multicolumn{5}{|c|}{ Adjusted OR b (95\%CI); p } \\
\hline & GG & TT & GT & TT+GT & GG & TT & GT & TT+GT & TT vs. (GG+GT) \\
\hline \multicolumn{10}{|l|}{ Sex } \\
\hline Male & $10 / 12$ & $598 / 762$ & $137 / 174$ & $735 / 936$ & 1.00 & $\begin{array}{c}1.062(0.46-2.48) \\
p: 0.889\end{array}$ & $\begin{array}{c}1.058(0.44-2.52) \\
p: 0.898\end{array}$ & $\begin{array}{c}1.061(0.46-2.47) \\
p: 0.890\end{array}$ & $\begin{array}{c}0.993(0.78-1.26) \\
p: 0.954\end{array}$ \\
\hline Female & $5 / 6$ & $210 / 291$ & $65 / 65$ & $275 / 356$ & 1.00 & $\begin{array}{c}1.155(0.35-3.83) \\
p: 1.000\end{array}$ & $\begin{array}{c}0.833(0.24-2.88) \\
p: 1.000\end{array}$ & $\begin{array}{c}1.079(0.33-3.57) \\
p: 1.000\end{array}$ & $\begin{array}{c}0.732(0.50-1.07) \\
p: 0.102\end{array}$ \\
\hline \multicolumn{10}{|l|}{ Age } \\
\hline$<63$ & $5 / 11$ & $373 / 506$ & $88 / 115$ & $461 / 621$ & 1.00 & $\begin{array}{c}0.617(0.21-1.79) \\
p: 0.450\end{array}$ & $\begin{array}{c}0.594(0.20-1.77) \\
p: 0.436\end{array}$ & $\begin{array}{c}0.612(0.21-1.77) \\
p: 0.450\end{array}$ & $\begin{array}{c}0.999(0.74-1.35) \\
p: 0.993\end{array}$ \\
\hline$\geq 63$ & $10 / 7$ & $435 / 547$ & $114 / 124$ & $549 / 671$ & 1.00 & $\begin{array}{c}1.796(0.68-4.76) \\
p: 0.232\end{array}$ & $\begin{array}{c}1.554(0.57-4.22) \\
p: 0.384\end{array}$ & $\begin{array}{c}1.746(0.66-4.62) \\
p: 0.255\end{array}$ & $\begin{array}{c}0.840(0.64-1.01) \\
p: 0.216\end{array}$ \\
\hline \multicolumn{10}{|l|}{ Smoking status } \\
\hline Never & $8 / 14$ & $450 / 777$ & $116 / 169$ & $566 / 946$ & 1.00 & $\begin{array}{c}0.987(0.41-2.37) \\
p: 0.976\end{array}$ & $\begin{array}{c}0.833(0.34-2.05) \\
p: 0.689\end{array}$ & $\begin{array}{c}0.955(0.40-2.29) \\
p: 0.918\end{array}$ & $\begin{array}{c}0.855(0.66-1.04) \\
p: 0.229\end{array}$ \\
\hline Ever & $7 / 4$ & $358 / 276$ & $86 / 70$ & $444 / 346$ & 1.00 & $\begin{array}{c}1.349(0.39-4.66) \\
p: 0.764\end{array}$ & $\begin{array}{c}1.424(0.40-5.06) \\
p: 0.756\end{array}$ & $\begin{array}{c}1.364(0.40-4.70) \\
p: 0.764\end{array}$ & $\begin{array}{c}1.032(0.73-1.46) \\
p: 0.857\end{array}$ \\
\hline \multicolumn{10}{|c|}{ Alcohol consumption } \\
\hline Never & $10 / 17$ & $556 / 978$ & $137 / 222$ & $693 / 1200$ & 1.00 & $\begin{array}{c}1.035(0.47-2.28) \\
p: 0.932\end{array}$ & $\begin{array}{c}0.953(0.42-2.14) \\
p: 0.908\end{array}$ & $\begin{array}{c}1.019(0.46-2.24) \\
p: 0.963\end{array}$ & $\begin{array}{c}0.924(0.73-1.16) \\
p: 0.503\end{array}$ \\
\hline Ever & $5 / 1$ & $252 / 75$ & $65 / 17$ & $317 / 92$ & 1.00 & $\begin{array}{c}1.488(0.17-12.94) \\
p: 1.000\end{array}$ & $\begin{array}{c}1.308(0.14-11.95) \\
p: 1.000\end{array}$ & $\begin{array}{c}1.451(0.17-12.58) \\
p: 1.000\end{array}$ & $\begin{array}{c}0.864(0.48-1.54) \\
p: 0.620\end{array}$ \\
\hline
\end{tabular}

${ }^{\text {a }}$ The genotyping success rate was $99.02 \%$ for rs $4646823 ;{ }^{\text {b }}$ Adjusted for age, sex, smoking status and alcohol consumption (besides stratified factors accordingly) in a logistic regression model; Fisher exact test was performed when $n \leq 5$; Bonferroni correction was performed to correct the $p$ value, bold values are statistically significant $(p<0.0125)$.

Table 7: Stratified analyses between $A L D H 3 B 2$ rs78402723 polymorphism and ESCC risk by sex, age, smoking status and alcohol consumption

\begin{tabular}{|c|c|c|c|c|c|c|c|c|c|}
\hline \multirow{2}{*}{ Variable } & \multicolumn{4}{|c|}{$\left(_{\text {case/control) }}{ }^{\mathrm{a}}\right.$} & \multicolumn{5}{|c|}{ Adjusted $\mathrm{OR}^{\mathrm{b}}(95 \% \mathrm{CI}) ; p$} \\
\hline & GG & $\mathbf{A A}$ & AG & $\mathbf{A A}+\mathbf{A G}$ & GG & AA & AG & $\mathbf{A A}+\mathbf{A G}$ & AA vs. (GG+AG) \\
\hline \multicolumn{10}{|l|}{ Sex } \\
\hline Male & $629 / 798$ & $4 / 7$ & $111 / 143$ & $115 / 150$ & 1.00 & $\begin{array}{c}1.379(0.40-4.73) \\
p: 0.764\end{array}$ & $\begin{array}{c}0.985(0.75-1.29) \\
p: 0.911\end{array}$ & $\begin{array}{c}0.973(0.75-1.27) ; \\
p: 0.837\end{array}$ & $\begin{array}{c}0.727(0.21-2.49) \\
p: 0.764\end{array}$ \\
\hline Female & $220 / 301$ & $5 / 3$ & $55 / 58$ & $60 / 61$ & 1.00 & $\begin{array}{c}0.439(0.10-1.85) \\
p: 0.294\end{array}$ & $\begin{array}{c}0.771(0.51-1.16) \\
p: 0.210\end{array}$ & $\begin{array}{c}0.743(0.50-1.11) \\
p: 0.141\end{array}$ & $\begin{array}{c}2.176(0.52-9.18) \\
p: 0.305\end{array}$ \\
\hline \multicolumn{10}{|l|}{ Age } \\
\hline$<63$ & $392 / 526$ & $4 / 8$ & $70 / 98$ & $74 / 106$ & 1.00 & $\begin{array}{c}1.490(0.45-4.99) \\
p: 0.572\end{array}$ & $\begin{array}{c}1.043(0.75-1.46) \\
p: 0.803\end{array}$ & $\begin{array}{c}1.068(0.77-1.48) ; \\
p: 0.693\end{array}$ & $\begin{array}{c}0.675(0.20-2.26) \\
p: 0.573\end{array}$ \\
\hline$\geq 63$ & $457 / 573$ & $5 / 2$ & $96 / 103$ & $101 / 105$ & 1.00 & $\begin{array}{c}0.319(0.06-1.65) \\
p: 0.252\end{array}$ & $\begin{array}{c}0.856(0.63-1.16) \\
p: 0.315\end{array}$ & $\begin{array}{c}0.829(0.62-1.12) \\
p: 0.220\end{array}$ & $\begin{array}{c}3.056(0.59-15.81) \\
p: 0.255\end{array}$ \\
\hline \multicolumn{10}{|c|}{ Smoking status } \\
\hline Never & $467 / 810$ & $6 / 7$ & $101 / 143$ & $107 / 150$ & 1.00 & $\begin{array}{c}0.673(0.23-2.01) \\
p: 0.476\end{array}$ & $\begin{array}{c}0.816(0.62-1.08) \\
p: 0.154\end{array}$ & $\begin{array}{c}0.808(0.62-1.06) ; \\
p: 0.126\end{array}$ & $\begin{array}{c}1.438(0.48-4.30) \\
p: 0.513\end{array}$ \\
\hline Ever & $382 / 289$ & $3 / 3$ & $65 / 58$ & $68 / 61$ & 1.00 & $\begin{array}{c}1.322(0.27-6.60) \\
p: 1.000\end{array}$ & $\begin{array}{c}1.179(0.80-1.73) \\
p: 0.401\end{array}$ & $\begin{array}{c}1.186(0.81-1.73) ; \\
p: 0.377\end{array}$ & $\begin{array}{c}0.776(0.16-3.87) \\
p: 1.000\end{array}$ \\
\hline \multicolumn{10}{|c|}{ Alcohol consumption } \\
\hline Never & $578 / 1023$ & $7 / 9$ & $118 / 185$ & $125 / 194$ & 1.00 & $\begin{array}{c}0.726(0.27-1.96) \\
p: 0.526\end{array}$ & $\begin{array}{c}0.886(0.69-1.14) \\
p: 0.346\end{array}$ & $\begin{array}{c}0.877(0.69-1.12) ; \\
p: 0.297\end{array}$ & $\begin{array}{c}1.350(0.50-3.64) \\
p: 0.552\end{array}$ \\
\hline Ever & $271 / 76$ & $2 / 1$ & $48 / 16$ & $50 / 17$ & 1.00 & $\begin{array}{c}1.783(0.16-19.93) ; \\
p: 0.527\end{array}$ & $\begin{array}{c}1.189(0.64-2.21) ; \\
p: 0.585\end{array}$ & $\begin{array}{c}1.212(0.66-2.22) ; \\
p: 0.533\end{array}$ & $\begin{array}{c}0.577(0.05-6.43) ; \\
p: 0.535\end{array}$ \\
\hline
\end{tabular}

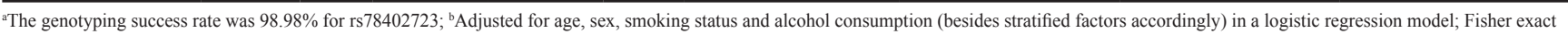
test was performed when $n \leq 5$; Bonferroni correction was performed to correct the $p$ value, bold values are statistically significant $(p<0.0125)$.

additional mutagenic species at concentrations attainable in vivo may underlie the carcinogenic effects [22]. Most of the acetaldehyde generated during alcohol metabolism in vivo is rapidly eliminated by aldehyde dehydrogenase $(\mathrm{ALDH})$, which renders $\mathrm{ALDH}$ an important role in carcinogen balancing and therefore carcinogenesis. In fact, there is ample evidence showed that subjects with an inactive form of ALDH2 (heterozygous for ALDH2 mutation) have an increased risk of developing various types of head and neck cancers as a consequent of intense exposure to acetaldehyde. Case-control studies of various Japanese drinking populations [23-29] and Chinese 
Table 8: Stratified analyses between $A L D H 3 B 2$ rs7947978 polymorphism and ESCC risk by sex, age, smoking status and alcohol consumption

\begin{tabular}{|c|c|c|c|c|c|c|c|c|c|}
\hline \multirow{2}{*}{ Variable } & \multicolumn{4}{|c|}{ (case/control) $^{a}$} & \multicolumn{5}{|c|}{ Adjusted OR ${ }^{\mathrm{b}}(95 \% \mathrm{CI}) ; p$} \\
\hline & $\mathrm{CC}$ & $\mathbf{A A}$ & CA & $\mathbf{A A}+\mathbf{C A}$ & $\mathrm{CC}$ & $\mathbf{A A}$ & CA & $\mathbf{A A}+\mathbf{C A}$ & AA vs. $(\mathrm{CC}+\mathrm{CA})$ \\
\hline \multicolumn{10}{|l|}{$\operatorname{Sex}$} \\
\hline Male & $595 / 762$ & $7 / 12$ & $143 / 174$ & $150 / 186$ & 1.00 & $\begin{array}{c}1.339(0.52-3.42) \\
p: 0.541\end{array}$ & $\begin{array}{c}0.950(0.74-1.22) \\
p: 0.683\end{array}$ & $\begin{array}{c}0.968(0.76-1.23) \\
p: 0.792\end{array}$ & $\begin{array}{c}0.740(0.29-1.89) \\
p: 0.527\end{array}$ \\
\hline Female & $209 / 292$ & $5 / 6$ & $66 / 64$ & $71 / 70$ & 1.00 & $\begin{array}{c}0.859(0.26-2.85) \\
p: 1.000\end{array}$ & $\begin{array}{c}0.694(0.47-1.02) \\
p: 0.064\end{array}$ & $\begin{array}{c}0.706(0.49-1.03) \\
p: 0.068\end{array}$ & $\begin{array}{c}1.079(0.33-3.57) \\
p: 1.000\end{array}$ \\
\hline \multicolumn{10}{|l|}{ Age } \\
\hline$<63$ & $372 / 507$ & $4 / 11$ & $90 / 114$ & $94 / 125$ & 1.00 & $\begin{array}{c}2.018(0.64-6.39) \\
p: 0.295\end{array}$ & $\begin{array}{c}0.929(0.68-1.26) \\
p: 0.640\end{array}$ & $\begin{array}{c}0.976(0.72-1.32) \\
p: 0.872\end{array}$ & $\begin{array}{c}0.489(0.16-1.55) \\
p: 0.295\end{array}$ \\
\hline$\geq 63$ & $432 / 547$ & $8 / 7$ & $119 / 124$ & $127 / 131$ & 1.00 & $\begin{array}{c}0.691(0.25-1.92) \\
p: 0.476\end{array}$ & $\begin{array}{c}0.823(0.62-1.09) \\
p: 0.174\end{array}$ & $\begin{array}{c}0.815(0.62-1.07) \\
p: 0.143\end{array}$ & $\begin{array}{c}1.392(0.50-3.86) \\
p: 0.524\end{array}$ \\
\hline \multicolumn{10}{|c|}{ Smoking status } \\
\hline Never & $447 / 778$ & $7 / 14$ & $120 / 168$ & $127 / 182$ & 1.00 & $\begin{array}{c}1.149(0.46-2.87) \\
p: 0.766\end{array}$ & $\begin{array}{c}0.824(0.62-1.05) \\
p: 0.102\end{array}$ & $\begin{array}{c}0.823(0.64-1.06) \\
p: 0.134\end{array}$ & $\begin{array}{c}0.834(0.34-2.08) \\
p: 0.697\end{array}$ \\
\hline Ever & $357 / 276$ & $5 / 4$ & $89 / 70$ & $94 / 74$ & 1.00 & $\begin{array}{c}1.035(0.28-3.89) \\
p: 1.000\end{array}$ & $\begin{array}{c}1.017(0.72-1.44) \\
p: 0.923\end{array}$ & $\begin{array}{c}1.018(0.72-1.44) \\
p: 0.918\end{array}$ & $\begin{array}{c}0.970(0.26-3.64) \\
p: 1.000\end{array}$ \\
\hline \multicolumn{10}{|c|}{ Alcohol consumption } \\
\hline Never & $552 / 979$ & $9 / 17$ & $142 / 221$ & $151 / 238$ & 1.00 & $\begin{array}{c}1.065(0.47-2.41) \\
p: 0.879\end{array}$ & $\begin{array}{c}0.878(0.69-1.11) \\
p: 0.276\end{array}$ & $\begin{array}{c}0.889(0.71-1.12) \\
p: 0.313\end{array}$ & $\begin{array}{c}0.915(0.41-2.07) \\
p: 0.831\end{array}$ \\
\hline Ever & $252 / 75$ & $3 / 1$ & $67 / 17$ & $70 / 18$ & 1.00 & $\begin{array}{c}1.120(0.12-10.93) \\
p: 1.000\end{array}$ & $\begin{array}{c}0.853(0.47-1.54) \\
p: 0.597\end{array}$ & $\begin{array}{c}0.864(0.48-1.54) \\
p: 0.620\end{array}$ & $\begin{array}{c}0.865(0.09-8.42) \\
p: 1.000\end{array}$ \\
\hline
\end{tabular}

Table 9: Stratified analyses between $A L D H 3 B 2$ rs866907 polymorphism and ESCC risk by sex, age, smoking status and alcohol consumption

\begin{tabular}{|c|c|c|c|c|c|c|c|c|c|}
\hline \multirow{2}{*}{ Variable } & \multicolumn{4}{|c|}{$\left(\right.$ case/control) ${ }^{\mathrm{a}}$} & \multicolumn{5}{|c|}{ Adjusted $\mathrm{OR}^{\mathrm{b}}(95 \% \mathrm{CI}) ; p$} \\
\hline & $\mathbf{A A}$ & GG & $\mathbf{A G}$ & $\mathbf{G G}+\mathbf{A G}$ & $\mathbf{A A}$ & GG & AG & $\mathbf{G G}+\mathbf{A G}$ & GG vs. $(A A+A G)$ \\
\hline \multicolumn{10}{|l|}{ Sex } \\
\hline Male & $7 / 12$ & $598 / 762$ & $140 / 174$ & $738 / 936$ & 1.00 & $\begin{array}{c}0.743(0.29-1.90) \\
p: 0.534\end{array}$ & $\begin{array}{c}0.725(0.28-1.89) \\
p: 0.509\end{array}$ & $\begin{array}{c}0.740(0.29-1.89) \\
p: 0.527\end{array}$ & $\begin{array}{c}0.993(0.78-1.26) \\
p: 0.954\end{array}$ \\
\hline Female & $5 / 6$ & $211 / 291$ & $64 / 65$ & $275 / 356$ & 1.00 & $\begin{array}{c}1.149(0.35-3.82) \\
p: 1.000\end{array}$ & $\begin{array}{c}0.846(0.25-2.91) \\
p: 1.000\end{array}$ & $\begin{array}{c}1.079(0.32-3.57) \\
p: 1.000\end{array}$ & $\begin{array}{c}0.746(0.51-1.09) \\
p: 0.126\end{array}$ \\
\hline \multicolumn{10}{|l|}{ Age } \\
\hline$<63$ & $4 / 11$ & $373 / 506$ & $89 / 115$ & $462 / 621$ & 1.00 & $\begin{array}{c}0.493(0.16-1.56) \\
p: 0.295\end{array}$ & $\begin{array}{c}0.470(0.15-1.53) \\
p: 0.281\end{array}$ & $\begin{array}{c}0.489(0.16-1.55) \\
p: 0.295\end{array}$ & $\begin{array}{c}0.999(0.74-1.35) \\
p: 0.993\end{array}$ \\
\hline$\geq 63$ & $8 / 7$ & $436 / 547$ & $115 / 124$ & $551 / 671$ & 1.00 & $\begin{array}{c}1.434(0.52-3.99) ; \\
p: 0.487\end{array}$ & $\begin{array}{c}1.232(0.43-3.51) \\
p: 0.695\end{array}$ & $\begin{array}{c}1.392(0.50-3.86) \\
p: 0.524\end{array}$ & $\begin{array}{c}0.849(0.64-1.12) \\
p: 0.245\end{array}$ \\
\hline \multicolumn{10}{|c|}{ Smoking status } \\
\hline Never & $7 / 14$ & $451 / 777$ & $116 / 169$ & $567 / 946$ & 1.00 & $\begin{array}{c}0.861(0.35-2.15) \\
p: 0.749\end{array}$ & $\begin{array}{c}0.728(0.29-1.86) \\
p: 0.506\end{array}$ & $\begin{array}{c}0.834(0.34-2.08) \\
p: 0.697\end{array}$ & $\begin{array}{c}0.864(0.67-1.12) \\
p: 0.262\end{array}$ \\
\hline Ever & $5 / 4$ & $358 / 276$ & $88 / 70$ & $446 / 346$ & 1.00 & $\begin{array}{c}0.964(0.26-3.62) \\
p: 1.000\end{array}$ & $\begin{array}{c}0.994(0.26-3.84) \\
p: 1.000\end{array}$ & $\begin{array}{c}0.970(0.26-3.64) \\
p: 1.000\end{array}$ & $\begin{array}{c}1.032(0.73-1.46) \\
p: 0.857\end{array}$ \\
\hline \multicolumn{10}{|c|}{$\begin{array}{l}\text { Alcohol } \\
\text { consumption }\end{array}$} \\
\hline Never & $9 / 17$ & $557 / 978$ & $137 / 222$ & $694 / 1200$ & 1.00 & $\begin{array}{c}0.930(0.41-2.10) \\
p: 0.860\end{array}$ & $\begin{array}{c}0.858(0.37-1.98) \\
p: 0.719\end{array}$ & $\begin{array}{c}0.915(0.41-2.07) \\
p: 0.831\end{array}$ & $\begin{array}{c}0.932(0.74-1.17) \\
p: 0.551\end{array}$ \\
\hline Ever & $3 / 1$ & $252 / 75$ & $67 / 17$ & $319 / 92$ & 1.00 & $\begin{array}{c}0.893(0.09-8.71) \\
p: 1.000\end{array}$ & $\begin{array}{c}0.761(0.07-7.78) \\
p: 1.000\end{array}$ & $\begin{array}{c}0.865(0.09-8.42) \\
p: 1.000\end{array}$ & $\begin{array}{c}0.864(0.48-1.54) \\
p: 0.620\end{array}$ \\
\hline
\end{tabular}

${ }^{a}$ The genotyping success rate was $99.02 \%$ for rs $866907{ }^{b}$ Adjusted for age, sex, smoking status and alcohol consumption (besides stratified factors accordingly) in a logistic regression model; Fisher exact test was performed when $n \leq 5$; Bonferroni correction was performed to correct the $p$ value, bold values are statistically significant $(p<0.0125)$.

alcoholics [30] have consistently reported that the inactive ALDH 2 encoded by the ALDH $2 * 1 / 2 * 2$ genotype is a strong risk factor for esophageal cancer. In resemblance with ALDH2, ALDH3 also plays a pivotal role in the alcohol metabolism, we thus hypothesize that ALDH3 family may be of potential relevance to carcinogenesis. 
Table 10: Stratified analyses between $A L D H 3 B 2$ rs9787887 polymorphism and ESCC risk by sex, age, smoking status and alcohol consumption

\begin{tabular}{|c|c|c|c|c|c|c|c|c|c|}
\hline \multirow{2}{*}{ Variable } & \multicolumn{4}{|c|}{$($ case/control) a } & \multicolumn{5}{|c|}{ Adjusted $\mathrm{OR}^{\mathrm{b}}(95 \% \mathrm{CI}) ; p$} \\
\hline & GG & $\mathbf{A A}$ & $\mathbf{A G}$ & $\mathbf{A G}+\mathbf{A A}$ & GG & $\mathbf{A A}$ & AG & $\mathbf{A G}+\mathbf{A A}$ & AA vs. (GG+AG) \\
\hline \multicolumn{10}{|l|}{ Sex } \\
\hline Male & $84 / 113$ & $319 / 420$ & $338 / 414$ & $657 / 834$ & 1.00 & $\begin{array}{c}0.979(0.71-1.35) \\
p: 0.894\end{array}$ & $\begin{array}{c}0.911(0.66-1.25) \\
p: 0.562\end{array}$ & $\begin{array}{c}0.944(0.70-1.27) \\
p: 0.705\end{array}$ & $\begin{array}{c}0.949(0.78-1.15) \\
p: 0.593\end{array}$ \\
\hline Female & $45 / 43$ & $119 / 168$ & $116 / 151$ & $235 / 319$ & 1.00 & $\begin{array}{c}1.477(0.92-2.39) \\
p: 0.110\end{array}$ & $\begin{array}{c}1.362(0.84-2.21) \\
p: 0.209\end{array}$ & $\begin{array}{c}1.421(0.91-2.23) \\
p: 0.126\end{array}$ & $\begin{array}{c}0.854(0.62-1.17) \\
p: 0.323\end{array}$ \\
\hline \multicolumn{10}{|l|}{ Age } \\
\hline$<63$ & $63 / 83$ & $203 / 287$ & $197 / 262$ & $400 / 549$ & 1.00 & $\begin{array}{c}1.158(0.80-1.67) \\
p: 0.433\end{array}$ & $\begin{array}{c}1.090(0.75-1.58) \\
p: 0.649\end{array}$ & $\begin{array}{c}1.124(0.80-1.59) \\
p: 0.506\end{array}$ & $\begin{array}{c}0.939(0.74-1.20) \\
p: 0.606\end{array}$ \\
\hline$\geq 63$ & $66 / 73$ & $235 / 301$ & $257 / 303$ & $492 / 604$ & 1.00 & $\begin{array}{c}1.158(0.80-1.68) \\
p: 0.442\end{array}$ & $\begin{array}{c}1.066(0.74-1.55) \\
p: 0.737\end{array}$ & $\begin{array}{c}1.110(0.78-1.58) \\
p: 0.563\end{array}$ & $\begin{array}{c}0.909(0.73-1.14) \\
p: 0.408\end{array}$ \\
\hline \multicolumn{10}{|c|}{ Smoking status } \\
\hline Never & $76 / 107$ & $245 / 430$ & $253 / 422$ & $498 / 852$ & 1.00 & $\begin{array}{c}1.247(0.89-1.74) \\
p: 0.194\end{array}$ & $\begin{array}{c}1.241(0.89-1.73) \\
p: 0.202\end{array}$ & $\begin{array}{c}1.215(0.89-1.66) \\
p: 0.223\end{array}$ & $\begin{array}{c}0.916(0.74-1.13) \\
p: 0.411\end{array}$ \\
\hline Ever & $53 / 49$ & $193 / 158$ & $201 / 143$ & $394 / 301$ & 1.00 & $\begin{array}{c}0.885(0.57-1.38) \\
p: 0.589\end{array}$ & $\begin{array}{c}0.770(0.49-1.20) \\
p: 0.246\end{array}$ & $\begin{array}{c}0.826(0.55-1.25) \\
p: 0.369\end{array}$ & $\begin{array}{c}0.923(0.70-1.22) \\
p: 0.579\end{array}$ \\
\hline \multicolumn{10}{|c|}{ Alcohol consumption } \\
\hline Never & $91 / 141$ & $305 / 552$ & $306 / 523$ & $611 / 1075$ & 1.00 & $\begin{array}{c}1.168(0.87-1.57) \\
p: 0.307\end{array}$ & $\begin{array}{c}1.103(0.82-1.49) \\
p: 0.520\end{array}$ & $\begin{array}{c}1.136(0.86-1.51) \\
p: 0.376\end{array}$ & $\begin{array}{c}0.924(0.77-1.11) \\
p: 0.409\end{array}$ \\
\hline Ever & $38 / 15$ & $133 / 36$ & $148 / 42$ & $281 / 78$ & 1.00 & $\begin{array}{c}0.686(0.34-1.38) \\
p: 0.291\end{array}$ & $\begin{array}{c}0.719(0.36-1.43) \\
p: 0.346\end{array}$ & $\begin{array}{c}0.703(0.37-1.35) \\
p: 0.285\end{array}$ & $\begin{array}{c}1.132(0.71-1.82) ; \\
p: 0.607\end{array}$ \\
\hline
\end{tabular}

Table 11: Linkage disequilibrium analyses of $A L D H 3 B 2$ rs34589365, rs3741172, rs4646823, rs78402723, rs7947978, rs866907 and rs9787887 in control group

\begin{tabular}{|c|c|c|c|c|c|c|}
\hline$D^{\prime}$ & rs3741172 & rs4646823 & rs78402723 & rs7947978 & rs866907 & rs9787887 \\
\hline rs34589365 & 0.991 & 0.993 & 0.992 & 0.993 & 0.993 & 0.977 \\
\hline rs3741172 & - & 1 & 1 & 0.995 & 1 & 1 \\
\hline rs 4646823 & - & - & 1 & 0.996 & 1 & 1 \\
\hline rs78402723 & - & - & - & 0.995 & 1 & 1 \\
\hline rs7947978 & - & - & - & - & 0.996 & 0.993 \\
\hline rs866907 & - & - & - & - & - & 1 \\
\hline$r^{2}$ & rs3741172 & rs4646823 & rs78402723 & rs7947978 & rs866907 & rs9787887 \\
\hline rs34589365 & 0.013 & 0.017 & 0.013 & 0.017 & 0.017 & 0.272 \\
\hline rs3741172 & - & 0.782 & 0.995 & 0.777 & 0.782 & 0.182 \\
\hline rs 4646823 & - & - & 0.786 & 0.988 & 1 & 0.233 \\
\hline rs78402723 & - & - & - & 0.781 & 0.786 & 0.183 \\
\hline rs7947978 & - & - & - & - & 0.988 & 0.229 \\
\hline rs866907 & - & - & - & - & - & 0.233 \\
\hline
\end{tabular}

In line with our speculation, overexpression of ALDH3 protects cells from 4-hydroxynonenal induced apoptosis, suggesting a functional relevance of ALDH3 in the carcinogenesis. On the other hand, many of the sphere cells and stem cells reported in different organs have recently been found to be associated with elevated ALDH1A1 enzyme activity [31-33]. ALDH1A1 expression or activity may be used with other cell surface markers to identify tumor-initiating cells in hepatocellular, prostate and breast solid carcinomas [34-36]. ALDH1A1 has also been detected to be associated with early metastasis and poor clinical outcome [33]. In addition, the ALDH enzymes also play a pivotal role in epithelial homeostasis. Deregulation of these enzymes is associated with multiple cancers [37- 
Table 12: Linkage disequilibrium analyses of $A L D H 3 B 2$ rs34589365, rs3741172, rs4646823, rs78402723, rs7947978, rs866907 and rs9787887 in case group

\begin{tabular}{lcccccc}
\hline \multicolumn{1}{c}{$\boldsymbol{D}^{\prime}$} & rs3741172 & rs4646823 & rs78402723 & rs7947978 & rs866907 & rs9787887 \\
\hline rs34589365 & $\mathbf{0 . 9 8 4}$ & $\mathbf{0 . 9 8 2}$ & $\mathbf{0 . 9 8 3}$ & $\mathbf{0 . 9 7 9}$ & $\mathbf{0 . 9 8 1}$ & $\mathbf{0 . 9 6 9}$ \\
rs3741172 & - & $\mathbf{1}$ & $\mathbf{1}$ & $\mathbf{1}$ & $\mathbf{1}$ & $\mathbf{0 . 9 8 9}$ \\
rs4646823 & - & - & $\mathbf{1}$ & $\mathbf{0 . 9 8 5}$ & $\mathbf{1}$ & $\mathbf{0 . 9 5 7}$ \\
rs78402723 & - & - & - & $\mathbf{1}$ & $\mathbf{1}$ & $\mathbf{1}$ \\
rs7947978 & - & - & - & - & $\mathbf{1}$ & $\mathbf{0 . 9 7 4}$ \\
rs866907 & - & - & - & - & - & $\mathbf{0 . 9 9 1}$ \\
\hline \multicolumn{1}{c}{ r2 } & rs3741172 & rs4646823 & rs78402723 & rs7947978 & rs866907 & rs9787887 \\
\hline rs34589365 & 0.014 & 0.017 & 0.013 & 0.017 & 0.017 & 0.248 \\
rs3741172 & - & $\mathbf{0 . 8 2 8}$ & $\mathbf{0 . 9 4 3}$ & $\mathbf{0 . 8 2 4}$ & $\mathbf{0 . 8 4 5}$ & 0.194 \\
rs4646823 & - & - & $\mathbf{0 . 7 8}$ & $\mathbf{0 . 9 6 6}$ & $\mathbf{0 . 9 8 1}$ & 0.219 \\
rs78402723 & - & - & - & $\mathbf{0 . 7 7 6}$ & $\mathbf{0 . 7 9 6}$ & 0.186 \\
rs7947978 & - & - & - & - & $\mathbf{0 . 9 7 6}$ & 0.228 \\
rs866907 & - & - & - & - & - & 0.231 \\
\hline
\end{tabular}

Table 13: $A L D H 3 B 2$ haplotype frequencies $(\%)$ in cases and controls and risk of ESCC

\begin{tabular}{|c|c|c|c|c|}
\hline Haplotypes & Case (freq) & $\begin{array}{c}\text { Control } \\
\text { (freq) }\end{array}$ & $\begin{array}{c}\text { Crude } \\
\text { OR(95\% CI) }\end{array}$ & $p$ \\
\hline$A L D H 3 B 2 \mathrm{~A}_{\mathrm{rs} 34589365} \mathrm{G}_{\mathrm{rs} 3741172} \mathrm{~T}_{\mathrm{rs} 4646823} \mathrm{G}_{\mathrm{rs} 78402723} \mathrm{C}_{\mathrm{rs} 7947978} \mathrm{G}_{\mathrm{rs} 866907} \mathrm{G}_{\mathrm{rs} 9777887}$ & $247(12.1)$ & $323(12.4)$ & $\begin{array}{c}1.007 \\
{[0.840 \sim 1.206]}\end{array}$ & 0.943 \\
\hline$A L D H 3 B 2 \mathrm{G}_{\mathrm{rs} 34589365} \mathrm{~A}_{\mathrm{rs} 3741172} \mathrm{G}_{\mathrm{rs} 4646823} \mathrm{~A}_{\mathrm{rs} 78402723} \mathrm{~A}_{\mathrm{rs} 5747978} \mathrm{~A}_{\mathrm{rs} 866907} \mathrm{G}_{\mathrm{rs} 9787887}$ & $184(9.0)$ & $219(8.4)$ & $\begin{array}{c}1.106 \\
{[0.898 \sim 1.363]}\end{array}$ & 0.344 \\
\hline$A L D H 3 B 2 \mathrm{G}_{\mathrm{rs} 34589365} \mathrm{G}_{\mathrm{r} s 3741172} \mathrm{~T}_{\mathrm{rs} 4646823} \mathrm{G}_{\mathrm{rs} 78402723} \mathrm{C}_{\mathrm{rs} 7947978} \mathrm{G}_{\mathrm{rs} 866907} \mathrm{~A}_{\mathrm{rs} 9787887}$ & $\begin{array}{c}1318 \\
(64.6)\end{array}$ & $1735(66.3)$ & 1.000 & 1.000 \\
\hline$A L D H 3 B 2 \mathrm{G}_{\mathrm{rs} 34589365} \mathrm{G}_{\mathrm{rr} 3741172} \mathrm{~T}_{\mathrm{r} 54646823} \mathrm{G}_{\mathrm{rs} 78402723} \mathrm{C}_{\mathrm{rs} 7947978} \mathrm{G}_{\mathrm{rs} 866907} \mathrm{G}_{\mathrm{rs} 9787887}$ & $235(11.5)$ & $279(10.6)$ & $\begin{array}{c}1.109 \\
{[0.919 \sim 1.338]}\end{array}$ & 0.281 \\
\hline
\end{tabular}

Haplotypes were composited by $A L D H 3 B 2$ rs34589365, rs3741172, rs4646823, rs78402723, rs7947978, rs866907, rs9787887. All those frequency $<3 \%$ were ignored in analysis, most common haplotype $A L D H 3 B 2 \mathrm{G}_{\mathrm{rs} 34589365} \mathrm{G}_{\mathrm{rs} 3741172}$ $\mathrm{T}_{\mathrm{r} 54646823} \mathrm{G}_{\mathrm{rs} 78402723} \mathrm{C}_{\mathrm{rs} 7947978} \mathrm{G}_{\mathrm{rs} 866907} \mathrm{~A}_{\mathrm{rs} 5787887}$ was selected as reference.

41]. In the current study, we demonstrated that there are no significant association between ALDH3B2 SNPs and risk of ESCC for the first time. More importantly, we have analyzed the interaction of the genetic background and environmental risk factors. These findings indicate that ALDH3B2 may not be the primary contributor to ESCC carcinogenesis. Considering the biological function of ALDH3B2, the evaluation with combined considerations based on both genetic and environmental factors would be much more precise and meaningful.

Cigarette smoking and alcohol drinking have emerged as well-known risk factors of ESCC. Although ALDH3B2 rs34589365, rs3741172, rs4646823, rs78402723, rs7947978, rs866907 and rs9787887 were not associated with the susceptibility of ESCC in the current study, the cigarette smoking rate and alcohol drinking rate were significantly higher in the ESCC cases, exemplifying the significance of interaction between the environmental and genetic risk factors in causing esophageal squamous carcinoma.

Our finding that there are more male than female subjects in the case group was in consistent with the comprehensive data recently published by the National Office for Cancer Prevention and Control as well as the National Cancer Center of China. The fact that smoking and alcohol drinking are far more prevalent in male subjects implicated the importance of these risk factors in carcinogenesis of ESCC.

One of the limitations of our previous studies investigating the association between SNPs and risk of 
ESCC was the sample size [12]. To overcome that, we have recruited a total of 2358 subjects including 1043 ESCC cases and 1315 controls in the current study. Yet, we do acknowledge there are still some limitations in this study. First, this study is limited by the sample sources, future studies designed and conduced in multiple ethnical populations and various geographic locations would be more convincing. Second, the lack of a validation cohort compromised the power of our study. Third, we are refrained by the lack of technical support to establish single nucleotide mutation cell/animal model, the actual biological function of these SNPs in esophageal carcinoma remains obscure, the underlying mechanisms are yet to be further dissected. Last but not least, the detailed information with regard to cancer metastasis and survival were not provided, the correlations between SNPs and outcomes have not been analyzed as this follow-up is still ongoing.

\section{MATERIALS AND METHODS}

\section{Ethics statement}

This hospital-based case-control study was approved by the Review Board of Jiangsu University (Zhenjiang, China). All subjects provided written informed consents. This study has complied with the World Medical Association Declaration of Helsinki with regard to ethical

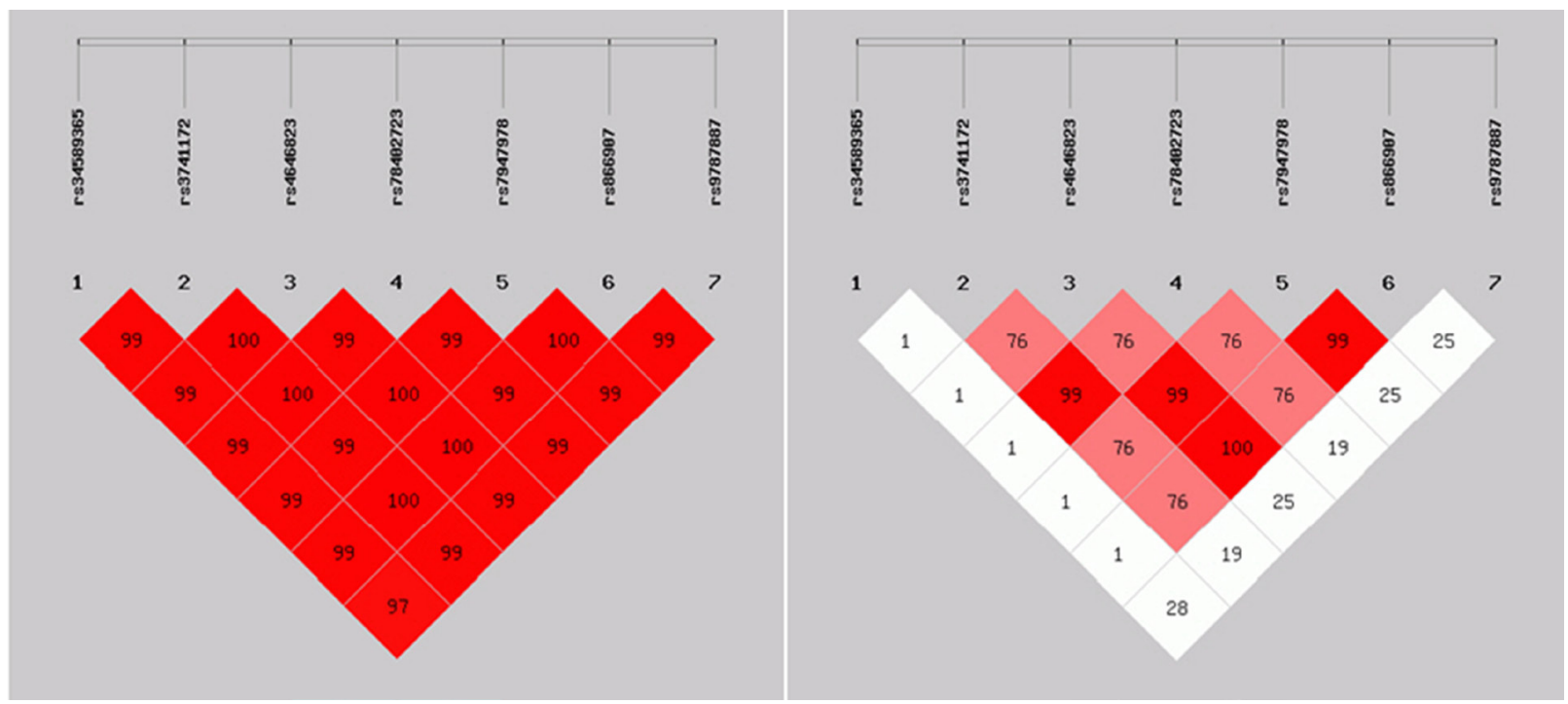

Figure 1: $D^{\prime}>0, r^{2}>0$ : There were linkage disequilibrium correlations among different loci; $D^{\prime}>70 \%, r^{2}>30 \%$. There were closer linkage disequilibrium correlations among different loci.

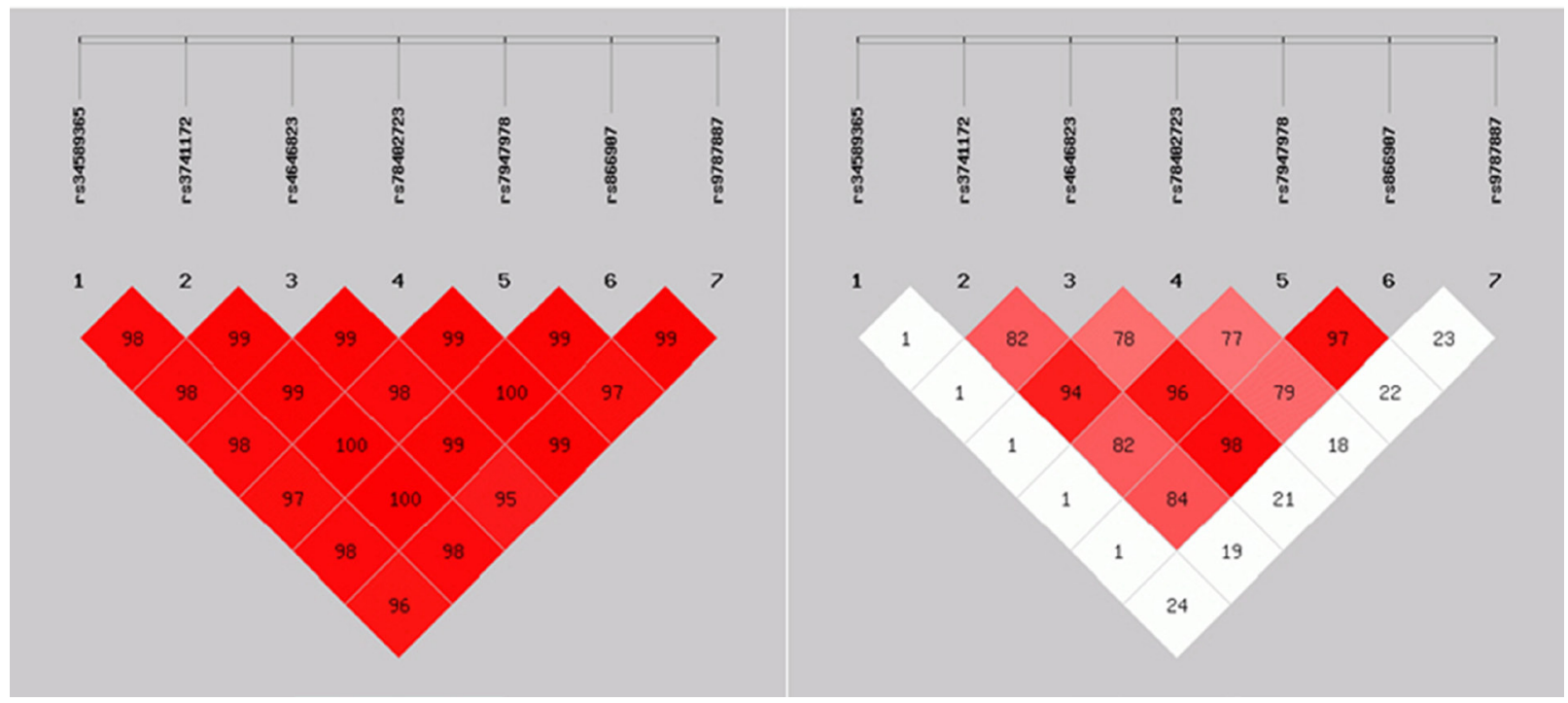

Figure 2: $D^{\prime}>0, r^{2}>0$ : There were linkage disequilibrium correlations among different loci; $D^{\prime}>70 \%(0.7), r^{2}>30 \%$ (0.3). There were closer linkage disequilibrium correlations among different loci. 
conduct of research involving human subjects and/or animals.

\section{Study populations}

A total of subjects consisting of 1043 ESCC cases and 1315 non-cancer controls frequency-matched to the cases regarding age and gender $(p=0.121$ and 0.880 , respectively) were enrolled in this study (Table 1). All patients and controls were consecutively recruited from the Affiliated People's Hospital of Jiangsu University (Zhenjiang, China) from October 2008 to January 2017. All cases of esophageal cancer were diagnosed as ESCC histologically. The exclusion criteria included cancer history, metastasized cancer or chemotherapy/radiotherapy history.

Each subject was individually questioned by experienced interviewers with a questionnaire to obtain information on demographic information and related risk factors (including alcohol consumption and cigarette smoking). After written informed consent was provided, two milliliters of venous blood were collected from each subject. The "Smokers" cohort included individuals who smoked one cigarette per day for more than one year. Subjects who had more than three alcoholic drinks a week for more than six months were included in the "Alcohol drinkers" cohort.

\section{Genomic DNA extraction, SNP selection and genotyping}

Genomic DNA was isolated from peripheral blood using QIAamp DNA Blood Mini Kit (Qiagen, Berlin, Germany) as reported [12]. Sample DNA were amplified by PCR according to the manufacturer's protocol. Gene polymorphisms were analyzed by the ligation detection reaction (LDR) method with technical support from Genesky Biotechnology Inc. (Shanghai, China). 10\% of the total samples were randomly selected for repeated analyses for quality control. Pilot linkage disequilibrium analyses were performed in the Chinese Han population to choose the SNP loci with moderate correlation, and tag SNPs were selected for further analyses.

\section{Statistical analyses}

Statistical analyses were conducted using SPSS 23.0 statistical package (SPCC Inc., Chicago, IL). Hardy-Weinberg equilibrium for genotypes was tested by goodness-of-fit $\chi^{2}$ in control group. Variations of demographic characteristics and genotypes of the $A L D H 3 B 2$ rs 34589365 , rs 3741172 , rs 4646823 , rs78402723, rs7947978, rs866907 and rs9787887 between the controls and cases were evaluated using the chi-square $\left(\chi^{2}\right)$ test to examine the statistical differences. The associations between these seven SNPs and risk of ESCC were analyzed by PLINK software (v1.07, available at http://zzz.bwh.harvard.edu/plink/download. shtml). Crude ORs and adjusted ORs when adjusting for age, sex, smoking and alcohol drinking status were also computed using logistic regression analyses. Bilateral probability tests were taken, $p$ value $<0.05$ was considered statistically significant.

\section{CONCLUSIONS}

The esophageal squamous carcinoma is associated with various factors including gene, environment and life-style. Our findings that $A L D H 3 B 2$ rs34589365, rs3741172, rs4646823, rs78402723, rs7947978, rs866907 and rs9787887 polymorphisms were not implicated with altered susceptibility of ESCC in different age, gender, cigarette smoking and alcohol drinking status, when interpreted with caution, could be helpful in evaluating the susceptibility to ESCC.

\section{ACKNOWLEDGMENTS}

We acknowledge the technical assistance from Genesky Biotechnologies Inc. (Shanghai, China).

\section{CONFLICTS OF INTEREST}

The authors declare no conflicts of interests.

\section{GRANT SUPPORT}

This study was supported by the National Natural Science Foundation of China (81300037, 81000028, 81370001, 81570031, 81101889, 81472332, 81341006); the Key Research and Development Program of Jiangsu Province (BE2016714); the Natural Science Foundation of Jiangsu Province (BK2010333, BK2011481); the "333" Elitist Training Program, Jiangsu, China (BRA2013135, BRA2017129); the "Six Talent Peaks" Training Program, Jiangsu, China (2015-WSN-117, 2014-WSN-078); the "Distinguished Medical Specialist" Program, Jiangsu, China; the "Innovative and Entrepreneurial Elite Team" Program (2016), Jiangsu, China, the "Young Medical Talents" Training Program (QNRC2016447), Jiangsu, China, the research funding of Zhongshan Hospital (2016ZSLC15), the Zhenjiang Social Development Program (SH2013039, SH2013045, SH2016048) and the Jiangsu University Clinical Medicine Science and Technology Development Program (JLY2010006).

\section{REFERENCES}

1. Fitzmaurice C, Dicker D, Pain A, Hamavid H, MoradiLakeh M, MacIntyre MF, Allen C, Hansen G, Woodbrook R, Wolfe C, Hamadeh RR, Moore A, Werdecker A, et al; 
Global Burden of Disease Cancer Collaboration. The Global Burden of Cancer 2013. JAMA Oncol. 2015; 1:505-27. https://doi.org/10.1001/jamaoncol.2015.0735.

2. Lagergren J, Smyth E, Cunningham D, Lagergren P. Oesophageal cancer. Lancet. 2017; 390:2383-2396. https:// doi.org/10.1016/S0140-6736(17)31462-9.

3. Xu Y, Yu X, Chen Q, Mao W. Neoadjuvant versus adjuvant treatment: which one is better for resectable esophageal squamous cell carcinoma? World J Surg Oncol. 2012; 10:173. https://doi.org/10.1186/1477-7819-10-173.

4. Gammon MD, Schoenberg JB, Ahsan H, Risch HA, Vaughan TL, Chow WH, Rotterdam H, West AB, Dubrow R, Stanford JL, Mayne ST, Farrow DC, Niwa $\mathrm{S}$, et al. Tobacco, alcohol, and socioeconomic status and adenocarcinomas of the esophagus and gastric cardia. J Natl Cancer Inst. 1997; 89:1277-84.

5. Lee $\mathrm{CH}, \mathrm{Wu} \mathrm{DC}$, Lee JM, Wu IC, Goan YG, Kao EL, Huang HL, Chan TF, Chou SH, Chou YP, Lee CY, Chen PS, Ho CK, et al. Carcinogenetic impact of alcohol intake on squamous cell carcinoma risk of the oesophagus in relation to tobacco smoking. Eur J Cancer. 2007; 43:1188-99. https://doi.org/10.1016/j.ejca.2007.01.039.

6. De Stefani E, Barrios E, Fierro L. Black (air-cured) and blond (flue-cured) tobacco and cancer risk. III: Oesophageal cancer. Eur J Cancer. 1993; 29A:763-6.

7. Enzinger PC, Mayer RJ. Esophageal cancer. N Engl J Med. 2003; 349:2241-52. https://doi.org/10.1056/NEJMra035010.

8. Brown LM, Hoover R, Silverman D, Baris D, Hayes R, Swanson GM, Schoenberg J, Greenberg R, Liff J, Schwartz A, Dosemeci M, Pottern L, Fraumeni JF Jr. Excess incidence of squamous cell esophageal cancer among US Black men: role of social class and other risk factors. Am J Epidemiol. 2001; 153:114-22.

9. Taylor PR, Qiao YL, Abnet CC, Dawsey SM, Yang CS, Gunter EW, Wang W, Blot WJ, Dong ZW, Mark SD. Prospective study of serum vitamin E levels and esophageal and gastric cancers. J Natl Cancer Inst. 2003; 95:1414-6.

10. Abnet CC, Lai B, Qiao YL, Vogt S, Luo XM, Taylor PR, Dong ZW, Mark SD, Dawsey SM. Zinc concentration in esophageal biopsy specimens measured by x-ray fluorescence and esophageal cancer risk. J Natl Cancer Inst. 2005; 97:301-6. https://doi.org/10.1093/jnci/dji042.

11. Matejcic M, Gunter MJ, Ferrari P. Alcohol Metabolism and Oesophageal Cancer: A Systematic Review of the Evidence. Carcinogenesis. 2017; 38:859-872. https://doi.org/10.1093/ carcin/bgx067.

12. Yin J, Wang L, Tang W, Wang X, Lv L, Shao A, Shi Y, Ding G, Chen S, Gu H. RANK rs1805034 T>C polymorphism is associated with susceptibility of esophageal cancer in a Chinese population. PLoS One. 2014; 9:e101705. https:// doi.org/10.1371/journal.pone.0101705.

13. IARC Working Group on the Evaluation of Carcinogenic Risks to Humans. Alcohol consumption and ethyl carbamate. IARC Monogr Eval Carcinog Risks Hum. 2010; 96:3-1383.
14. IARC Working Group on the Evaluation of Carcinogenic Risks to Humans. Personal habits and indoor combustions. Volume 100 E. A review of human carcinogens. IARC Monogr Eval Carcinog Risks Hum. 2012; 100:1-538.

15. Secretan B, Straif K, Baan R, Grosse Y, El Ghissassi F, Bouvard V, Benbrahim-Tallaa L, Guha N, Freeman C, Galichet L, Cogliano V; WHO International Agency for Research on Cancer Monograph Working Group. A review of human carcinogens--Part E: tobacco, areca nut, alcohol, coal smoke, and salted fish. Lancet Oncol. 2009; 10:1033-4.

16. Zakhari S. Overview: how is alcohol metabolized by the body? Alcohol Res Health. 2006; 29:245-54.

17. Ji Y, Li X, Li Y, Zhong Y, Cao J, Xu R, Wang J, Zhou F, Li X, Yu D, Wen JG, Goscinski MA, Zhang M, et al. Aldehyde Dehydrogenase-1 Expression Predicts Unfavorable Outcomes in Patients with Esophageal Squamous Cell Carcinoma. Anticancer Res. 2016; 36:343-9.

18. Crabb DW, Edenberg HJ, Bosron WF, Li TK. Genotypes for aldehyde dehydrogenase deficiency and alcohol sensitivity. The inactive ALDH2(2) allele is dominant. J Clin Invest. 1989; 83:314-6. https://doi.org/10.1172/JCI113875.

19. Liu K, Song G, Zhu X, Yang X, Shen Y, Wang W, Shi G, Li Q, Duan Y, Zhao Y, Feng G. Association between ALDH2 Glu487Lys polymorphism and the risk of esophageal cancer. Medicine (Baltimore). 2017; 96:e6111. https://doi. org/10.1097/MD.0000000000006111.

20. Yukawa Y, Muto M, Hori K, Nagayoshi H, Yokoyama A, Chiba T, Matsuda T. Combination of ADH1B*2/ ALDH2*2 polymorphisms alters acetaldehyde-derived DNA damage in the blood of Japanese alcoholics. Cancer Sci. 2012; 103:1651-5. https://doi.org/10.1111/j.13497006.2012.02360.x.

21. Ma WJ, Lv GD, Zheng ST, Huang CG, Liu Q, Wang X, Lin RY, Sheyhidin I, Lu XM. DNA polymorphism and risk of esophageal squamous cell carcinoma in a population of North Xinjiang, China. World J Gastroenterol. 2010; 16:641-7.

22. Marietta C, Thompson LH, Lamerdin JE, Brooks PJ. Acetaldehyde stimulates FANCD2 monoubiquitination, H2AX phosphorylation, and BRCA1 phosphorylation in human cells in vitro: implications for alcohol-related carcinogenesis. Mutat Res. 2009; 664:77-83. https://doi. org/10.1016/j.mrfmmm.2009.03.011.

23. Yokoyama A, Muramatsu T, Ohmori T, Higuchi S, Hayashida M, Ishii H. Esophageal cancer and aldehyde dehydrogenase-2 genotypes in Japanese males. Cancer Epidemiol Biomarkers Prev. 1996; 5:99-102.

24. Hori H, Kawano T, Endo M, Yuasa Y. Genetic polymorphisms of tobacco- and alcohol-related metabolizing enzymes and human esophageal squamous cell carcinoma susceptibility. J Clin Gastroenterol. 1997; 25:568-75.

25. Yokoyama A, Muramatsu T, Ohmori T, Yokoyama T, Okuyama K, Takahashi H, Hasegawa Y, Higuchi S, 
Maruyama K, Shirakura K, Ishii H. Alcohol-related cancers and aldehyde dehydrogenase-2 in Japanese alcoholics. Carcinogenesis. 1998; 19:1383-7.

26. Tanabe H, Ohhira M, Ohtsubo T, Watari J, Yokota K, Kohgo Y. Genetic polymorphism of aldehyde dehydrogenase 2 in patients with upper aerodigestive tract cancer. Alcohol Clin Exp Res. 1999; 23:17S-20S.

27. Yokoyama A, Muramatsu T, Omori T, Yokoyama T, Matsushita S, Higuchi S, Maruyama K, Ishii H. Alcohol and aldehyde dehydrogenase gene polymorphisms and oropharyngolaryngeal, esophageal and stomach cancers in Japanese alcoholics. Carcinogenesis. 2001; 22:433-9.

28. Matsuo K, Hamajima N, Shinoda M, Hatooka S, Inoue M, Takezaki T, Tajima K. Gene-environment interaction between an aldehyde dehydrogenase-2 (ALDH2) polymorphism and alcohol consumption for the risk of esophageal cancer. Carcinogenesis. 2001; 22:913-6.

29. Itoga S, Nomura F, Makino Y, Tomonaga T, Shimada H, Ochiai T, Iizasa T, Baba M, Fujisawa T, Harada S. Tandem repeat polymorphism of the CYP2E1 gene: an association study with esophageal cancer and lung cancer. Alcohol Clin Exp Res. 2002; 26:15S-9S. https://doi.org/10.1097/01. ALC.0000026828.13868.B5.

30. Chao YC, Wang LS, Hsieh TY, Chu CW, Chang FY, Chu HC. Chinese alcoholic patients with esophageal cancer are genetically different from alcoholics with acute pancreatitis and liver cirrhosis. Am J Gastroenterol. 2000; 95:2958-64. https://doi.org/10.1111/j.1572-0241.2000.02328.x.

31. Charafe-Jauffret E, Ginestier C, Iovino F, Wicinski J, Cervera N, Finetti P, Hur MH, Diebel ME, Monville F, Dutcher J, Brown M, Viens P, Xerri L, et al. Breast cancer cell lines contain functional cancer stem cells with metastatic capacity and a distinct molecular signature. Cancer Res. 2009; 69:1302-13. https://doi. org/10.1158/0008-5472.CAN-08-2741.

32. Carpentino JE, Hynes MJ, Appelman HD, Zheng T, Steindler DA, Scott EW, Huang EH. Aldehyde dehydrogenase-expressing colon stem cells contribute to tumorigenesis in the transition from colitis to cancer. Cancer Res. 2009; 69:8208-15. https://doi.org/10.1158/0008-5472. CAN-09-1132.

33. Charafe-Jauffret E, Ginestier C, Iovino F, Tarpin C, Diebel M, Esterni B, Houvenaeghel G, Extra JM, Bertucci F, Jacquemier J, Xerri L, Dontu G, Stassi G, et al. Aldehyde dehydrogenase 1-positive cancer stem cells mediate metastasis and poor clinical outcome in inflammatory breast cancer. Clin Cancer Res. 2010; 16:45-55. https://doi. org/10.1158/1078-0432.CCR-09-1630.
34. Colombo F, Baldan F, Mazzucchelli S, Martin-Padura I, Marighetti P, Cattaneo A, Foglieni B, Spreafico M, Guerneri S, Baccarin M, Bertolini F, Rossi G, Mazzaferro V, et al. Evidence of distinct tumour-propagating cell populations with different properties in primary human hepatocellular carcinoma. PLoS One. 2011; 6:e21369. https://doi. org/10.1371/journal.pone.0021369.

35. van den Hoogen C, van der Horst G, Cheung H, Buijs JT, Lippitt JM, Guzman-Ramirez N, Hamdy FC, Eaton CL, Thalmann GN, Cecchini MG, Pelger RC, van der Pluijm G. High aldehyde dehydrogenase activity identifies tumor-initiating and metastasis-initiating cells in human prostate cancer. Cancer Res. 2010; 70:5163-73. https://doi. org/10.1158/0008-5472.CAN-09-3806.

36. Buijs JT, van der Horst G, van den Hoogen C, Cheung H, de Rooij B, Kroon J, Petersen M, van Overveld PG, Pelger $\mathrm{RC}$, van der Pluijm G. The BMP2/7 heterodimer inhibits the human breast cancer stem cell subpopulation and bone metastases formation. Oncogene. 2012; 31:2164-74. https:// doi.org/10.1038/onc.2011.400.

37. van den Hoogen C, van der Horst G, Cheung H, Buijs JT, Pelger RC, van der Pluijm G. The aldehyde dehydrogenase enzyme 7A1 is functionally involved in prostate cancer bone metastasis. Clin Exp Metastasis. 2011; 28:615-25. https://doi.org/10.1007/s10585-011-9395-7.

38. Marcato P, Dean CA, Pan D, Araslanova R, Gillis M, Joshi M, Helyer L, Pan L, Leidal A, Gujar S, Giacomantonio CA, Lee PW. Aldehyde dehydrogenase activity of breast cancer stem cells is primarily due to isoform ALDH1A3 and its expression is predictive of metastasis. Stem Cells. 2011; 29:32-45. https://doi.org/10.1002/stem.563.

39. Marchitti SA, Orlicky DJ, Brocker C, Vasiliou V. Aldehyde dehydrogenase 3B1 (ALDH3B1): immunohistochemical tissue distribution and cellular-specific localization in normal and cancerous human tissues. J Histochem Cytochem. 2010; 58:765-83. https://doi.org/10.1369/ jhc.2009.95577310.1369/jhc.2010.955773.

40. Marcato P, Dean CA, Giacomantonio CA, Lee PW. Aldehyde dehydrogenase: its role as a cancer stem cell marker comes down to the specific isoform. Cell Cycle. 2011; 10:1378-84. https://doi.org/10.4161/cc.10.9.15486.

41. Zhang Q, Taguchi A, Schliekelman M, Wong CH, Chin A, Kuick R, Misek DE, Hanash S. Comprehensive proteomic profiling of aldehyde dehydrogenases in lung adenocarcinoma cell lines. Int J Proteomics. 2011; 2011:145010. https://doi.org/10.1155/2011/145010. 